\title{
Electron cloud trapping in combined function dipole magnets
}

\section{Sergey A. Antipov ${ }^{1}$}

The University of Chicago

5720 S. Ellis ave, Chicago, IL, USA

E-mail: antipoveuchicago.edu

\section{Sergei Nagaitsev, , USA}

Fermilab

Pine st and Kirk rd, Batavia, IL, USA

E-mail: nsergeiefnal.gov

Electron cloud can lead to a fast instability in intense proton and positron beams in circular accelerators. We demonstrate that electron cloud can be trapped in a combined function magnet. We present the results of analytical estimates, and compare them to numerical simulations of electron cloud formation. In a combined function magnet the electron cloud is located at the beam center and the ratio of trapped particles can be as high as $1 \%$. Since the process of electron cloud build-up is exponential, once trapped this amount of electrons significantly decreases the time of build-up on the next revolution. The trapping creates a mechanism for the beam to act on itself on the next turn and can lead to a head-tail instability.

38th International Conference on High Energy Physics

3-10 August 2016

Chicago, USA

\section{${ }^{1}$ Speaker}




\section{Introduction}

In 2014 a fast transverse instability was observed in the proton beam of the Fermilab Recycler. The instability acts only in the horizontal plane and typically develops in about 20-30 revolutions. It also has the unusual feature of selectively impacting the first batch above the threshold intensity of $\sim 4 * 10^{10}$ protons per bunch (Fig. 1). These peculiar features suggest that a possible cause of the instability is electron cloud. Earlier studies [1] indicated the presence of electron cloud in the ring and suggested the possibility of its trapping in Recycler combined function magnets.

In a combined function dipole the electrons of the cloud move along the vertical field lines. This motion conserves their energy $E$ and magnetic moment

$$
\mu=\frac{m v_{\perp}^{2}}{2 B}=\text { const }
$$

where $v_{\perp}$ is the component of the velocity normal to the magnetic field $B$. As an electron moves closer to a magnet pole it sees a higher $B$ (Fig. 1) and it can reflect back if

$$
E-\mu B=0
$$

Alternatively, the electron will reflect back at the point of maximum magnetic field if the angle between the electron's velocity and the field lines is greater than:

$$
\theta>\theta_{\max }=\cos ^{-1}\left(\sqrt{B_{0} / B_{\max }}\right) .
$$

Particles with angles $\theta_{\max }<\theta \leq \pi / 2$ are trapped by magnetic field. For Recycler magnets (Table 1), Eq. (3) gives a capture of $\sim 10^{-2}$ particles of electron cloud, assuming uniform distribution.

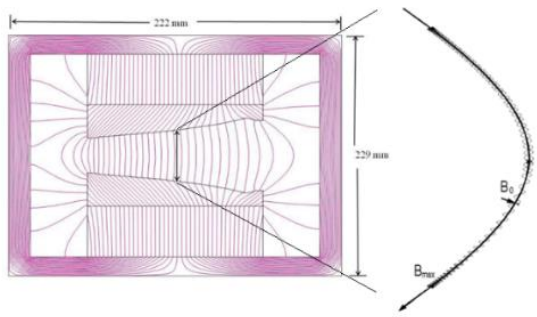

Figure 1: Electron cloud can get trapped by magnetic field of a combined function magnet.

\section{Simplified analytical model of trapping}

\subsection{Electron capture by the field}

Let us look at the process of electron cloud trapping in more detail and consider the last two bunches of the batch. The first bunch kicks the electrons of the cloud, created by the batch. With an energy of the order of $100 \mathrm{eV}$ the electrons drift along the magnetic field in the vacuum chamber, finally reaching its walls and producing secondary electrons with the energies of a few eV [2]. In the absence of the beam these secondary electrons would eventually reach the aperture and die. But the next proton bunch can stop a fraction of the secondaries, increasing the angle $\theta$ above the critical value Eq. (3) (Fig. 2). These electrons will remain trapped in the magnetic field after the beam is gone.

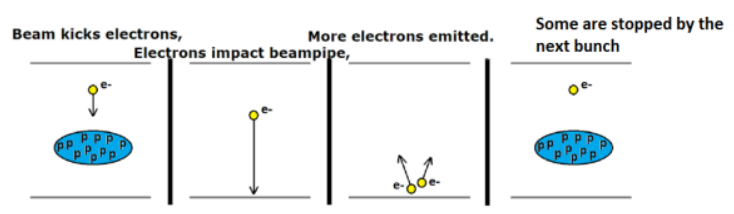

Figure 2: The first bunch kicks the cloud, creating secondary electrons, the second bunch stops some of them.

Thanks to the strong magnetic field the motion of an electron is essentially a $1 \mathrm{D}$ problem that can be easily solved. Initially, the secondaries start at the wall of the vacuum chamber, and have some distribution of velocities $\mathbf{v}=\left(v_{y}, v_{\perp}\right)$. During one RF period the electrons travel $\Delta y=v_{y} \tau_{R F}$ before receiving a kick from the next proton bunch. Assuming a transverse waterbag and longitudinal Gaussian beam profile and neglecting the self space-charge of the electron cloud the equation of motion becomes 


$$
\begin{aligned}
& \left\{\begin{array}{l}
y^{\prime \prime}+C \cdot y \exp \left(-t^{2} \mathrm{c}^{2} / 2 \sigma_{s}^{2}\right)=0, y<r_{0} \\
\mathrm{y}^{\prime \prime}+C \cdot \frac{r_{0}^{2}}{y} \exp \left(-\mathrm{t}^{2} \mathrm{c}^{2} / 2 \sigma_{s}^{2}\right)=0, y \geq r_{0}
\end{array}\right. \\
& C=\frac{q_{e}^{2} N_{b}}{m_{e} r_{0}^{2} \sqrt{2 \pi \sigma_{s}^{2}}},
\end{aligned}
$$

where $r_{0}$ and $\sigma_{s}$ are the transverse and longitudinal bunch sizes and $N_{b}$ - bunch population.

The system in Eq. (4) has to be solved with the initial condition $y(t=0)=-A / 2$ and a boundary condition $|y(t>0)|<A / 2$, where $\mathrm{A}$ is the physical aperture. Assuming an initial distribution from [2] one can estimate the fraction of the electron cloud that falls within the trapping cone defined by Eq. (3) and stays trapped by the field after the beam is gone. For Recycler magnets this ratio can be as high as a few percent for low beam intensities. At the current operational intensity of $5 \times 10^{10} \mathrm{ppb}$ about $1 \%$ of particles are trapped, and at higher intensities this amount gets smaller (Fig. 3). This trapped cloud will be present in the magnet on the next revolution.

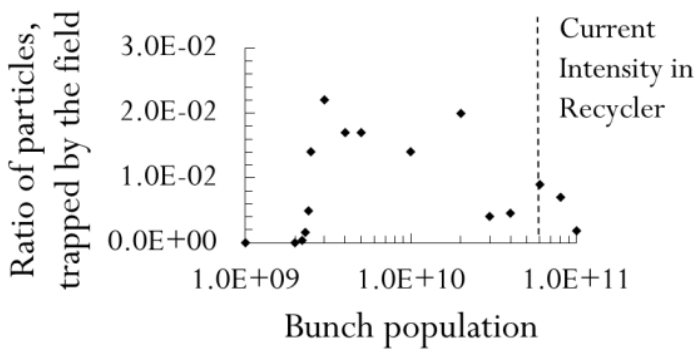

Figure 3: About $1 \%$ of electrons are trapped at the current beam intensity in Recycler. This fraction reduces for higher intensities.

\section{Numerical simulation of electron cloud build-up}

We simulated electron cloud build-up over multiple revolutions in a Recycler dipole using the PEI code [3]. For a pure dipole field, the cloud rapidly builds up during the passage of the bunch train and then decays back to the initial ionization electron density in about $300 \mathrm{RF}$ buckets, or $\sim 6 \mu \mathrm{s}$ (Fig. 4). When the field gradient is added, up to $1 \%$ of the electron cloud stays trapped, increasing the initial density on the next revolution. The final density, which the cloud reaches after $\sim 10$ revolutions, is two orders of magnitude greater than in the pure dipole case (Fig. 4). The resulting cloud distribution is a stripe along the magnetic field lines, with higher particle density being closer to the walls of the vacuum chamber (Fig. 5). The width of the stripe is approximately equal to the size of the beam and its intensity increases from turn to turn as the cloud builds up.

At lower densities $\sim 10^{-2}$ of particles are trapped, which agrees with the analytic estimate (Fig. 3); as the density of electron cloud increases the trapping ratio goes down to $\sim 10^{-3}$, probably due to the space charge of electron cloud.

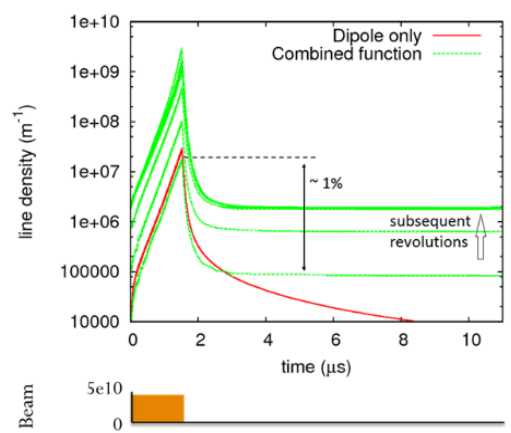

Figure 4: In a combined function magnet the electron cloud accumulates over many revolutions, reaching much higher line density, than in a dipole. 

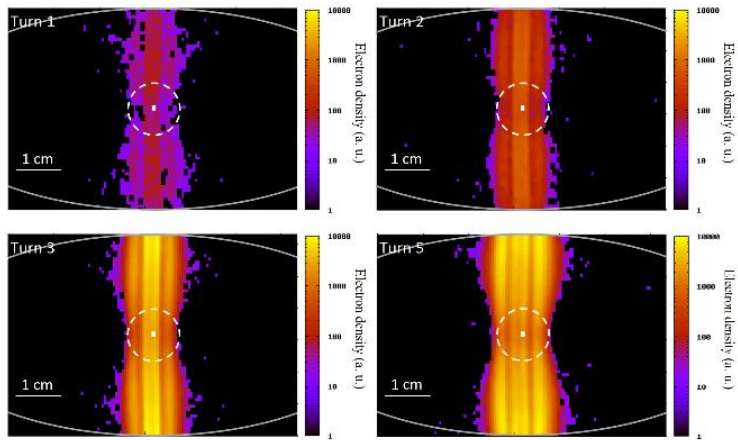

Figure 5: Electron cloud forms a stripe inside the vacuum chamber and its intensity increases with the number of turns. Its horizontal position - beam center (white dot). White circles represent $2 \mathrm{rms}$ beam size.

Table 1: Recycler parameters for simulation in PEI

\begin{tabular}{llll}
\hline \hline Beam energy & $8 \mathrm{GeV}$ & Secondary emission yield $2.1 @ 250 \mathrm{eV}$ \\
Ring circumference & $3.3 \mathrm{~km}$ & Density of ionization $\mathrm{e}^{-}$ & $10^{4} \mathrm{~m}^{-1}$ (at $\left.10^{-8} \mathrm{Torr}\right)$ \\
Batch structure & 80 bunches, $5 \mathrm{e} 10 \mathrm{p}$ & B-field and its gradient & $1.38 \mathrm{kG}, 3.4 \mathrm{kG} / \mathrm{m}$ \\
Tunes: $\mathrm{x}, \mathrm{y}, \mathrm{z}$ & $25.45,24.40,0.003$ & Magnet length & $5 \mathrm{~m}$ \\
RF harmonic, period & $588 ; 18.9 \mathrm{~ns}$ & Beampipe & Elliptical, $100 \mathrm{x} 44 \mathrm{~mm}$ \\
rms bunch size: $\mathrm{x}, \mathrm{y}, \mathrm{z}$ & $0.3,0.3,60 \mathrm{~cm}$ & & \\
\hline
\end{tabular}

\section{Conclusion}

Combined function magnets are widely used in the present day machines. Because of the gradient of the magnetic field (which provides the focusing) the electron cloud can be trapped in the magnetic field of such magnets. These trapped particles make it possible for the cloud to accumulate over multiple revolutions, possibly leading to a fast transverse instability.

We have created an analytical model that allows the estimation of the amount of the cloud captured in the magnet. We have shown that up to $1 \%$ of the electron cloud can be trapped in the magnetic field of combined function magnets of FNAL Recycler. This fraction of trapped particles will go down for higher intensities in Recycler.

Numerical simulation in PEI agrees with the analytical estimate and confirms that the trapping significantly affects the density of the electron could. It allows the cloud to accumulate over multiple revolutions reaching a density much higher than in a pure dipole. For the parameters of Fermilab Recycler with one batch of normal intensity the cloud reaches $\sim 10^{9} \mathrm{~m}^{-1}$ in a combined function magnet compared to $\sim 10^{7} \mathrm{~m}^{-}$ ${ }^{1}$ in a dipole of the same field strength.

\section{Acknowledgment}

The authors are grateful to K. Ohmi (KEK) for his help with PEI code. Fermilab is operated by Fermi Research Alliance, LLC under Contract No. DE-AC02-07CH11359 with the United States Department of Energy.

\section{References}

[1] J. Eldred, et al., in Proc. HB' 14, pp. 419-427

[2] G. Iadarola PhD Thesis, CERN-THESIS-2014-047

[3] K. Ohmi, CERN Rep. CERN-2013-002, pp.219-224 